\title{
Role of nitric oxide synthase on brain GABA transaminase activity and GABA levels
}

\author{
LOURDES A. VEGA RASGADO ${ }^{1 *}$ \\ GUILLERMO CEBALLOS REYES ${ }^{2}$ \\ FERNANDO VEGA DÍAZ ${ }^{1}$ \\ ${ }^{1}$ Laboratorio de Neuroquímica \\ Departamento de Bioquímica \\ Escuela Nacional de Ciencias Biológicas \\ Instituto Politécnico Nacional \\ Carpio y Plan de Ayala S/N, Colonia Casco \\ de Santo Tomás, C.P. 11340 \\ Ciudad de México, México \\ ${ }^{2}$ Laboratorio de Investigación Integral \\ Cardiometabólica \\ Sección de Posgrado e Investigación \\ Escuela Superior de Medicina \\ Instituto Politécnico Nacional. Plan de San \\ Luis y Díaz Mirón \\ Colonia Casco de Santo Tomás, C.P. 11340 \\ Ciudad de México, México \\ Accepted February 12, 2018 \\ Published online March 16, 2018
}

\begin{abstract}
In an attempt to clarify the controversial role of nitric oxide (NO) in seizures, the effects of $\mathrm{NO}$ on brain GABA transaminase (GABA-T) activity and GABA levels were investigated. To this aim, the effects of the substrate (L-arginine) and inhibitors ( $\mathrm{N \omega}$-nitro-Larginine methyl ester, 7-nitroindazole) of NO synthase (NOS) on GABA-T activity and GABA levels in vitro and ex vivo were analyzed. In vitro NO diminished GABA-T activity and increased GABA. Ex vivo NO modified GABA-T activity and GABA levels biphasically. Inhibition of endothelial and neuronal NOS (eNOS and nNOS) had opposite effects on GABA-T activity and GABA levels, even during seizures induced by pentylenetetrazole. Different effects of NO on GABA-T activity and on GABA levels, depending on the NOS isoform involved, may explain its contradictory role in seizures, the endothelial NOS acting as an anticonvulsant and the neuronal NOS as a proconvulsant. nNOS inhibitors may represent a new generation of antiepileptics.
\end{abstract}

Keywords: nitric oxide, GABA-transaminase, GABA, seizures

Nitric oxide (NO) is a freely diffusible gas synthesized from the oxidation of L-arginine by one of the isoforms of the enzyme NO synthase (NOS): neuronal (nNOS), endothelial (eNOS) and inducible (iNOS). In the brain, NO acts both as a second messenger and as a neurotransmitter/neuromodulator, and influences several physiological functions $(1,2)$ such as: interneuronal communication, synaptic plasticity, memory, intracellular signal transmission and mediator release (3). Also, it has been proposed that NO can induce pathologies that can lead to different neurological disorders such as ischemia, stroke and epileptiform seizures (2).

The role of NO in epileptogenesis has been examined in a number of ex vivo and in vitro studies; however, the results are still contradictory. In the present state of knowledge,

\footnotetext{
*Correspondence; e-mail: lourdes_vega_rasgado@hotmail.com
} 
the only reasonable conclusion is that $\mathrm{NO}$ behaves as a neuromodulador with dual - proconvulsive or anticonvulsive - action (4).

The main intracellular effect of NO in the central nervous system (CNS) is the activation of soluble guanylate cyclase, leading to the synthesis of cyclic guanosine monophosphate (cGMP) (5). As an example of the importance of the NO-cGMP pathway, its involvement in the neuroprotective effect of hesperidin in mice could be mentioned (6). However, $\mathrm{NO}$ also evokes the release of neurotransmitters including acetylcholine, catecholamines and neuroactive amino acids such as gamma aminobutyric acid (GABA) (7).

Seizures may appear when the balance between excitatory and inhibitory impulses in the brain is perturbed. GABA is considered to be the main inhibitory neurotransmitter of the CNS that counterbalances neuronal excitation. One of the strategies to prevent and/ or control convulsions is to increase GABA brain levels through inhibition of GABA transaminase (GABA-T, EC 2.6.1.19), the enzyme responsible for its degradation. Based on this principle, anticonvulsant drugs such as amino oxyacetic acid (AAOA) and antiepileptic drugs such as valproic acid (8), vigabatrin (9) and ethanolamine-O-sulfate (10) were designed.

Stimulation of glutamate receptors, mainly of the $N$-methyl-D-aspartate (NMDA) type, is a consequence of the increase in cGMP caused by NO. It has been reported that NMDA receptors may contribute to both anti- and pro-convulsant effects of D-penicillamine (11). NMDA receptor activation induces release of GABA from the cerebral cortical and striatal neurons (12-14). In addition, it has been reported that the increase in NO concentration is associated with the release of GABA in cerebral cortex (7), hippocampus (15) and striatum (16-18); however, the mechanisms still remain unclear. As an example, it is not clear why moderate and high increases of NO levels had opposite effects on GABA-T activity (18).

Considering the foregoing and the widespread role of NO in the nervous system, we conducted an in vitro and ex vivo study in order to explore the possible factors influencing the effect of NO on GABA-T activity. To this aim, the effects of promotion of NO synthesis induced by L-arginine (L-Arg) on GABA-T activity and GABA levels in whole mouse brain were investigated. The effect of NO synthesis inhibition on the described parameters was also studied using $N \omega$-nitro-L-arginine-methyl ester (L-NAME), an unspecific inhibitor of NOS. Since nNOS is distributed in several sensory pathways $(19,20)$, 7-nitroindazole (7$\mathrm{NI})$, a specific inhibitor of nNOS that administrated in doses up to $80 \mathrm{mg} \mathrm{kg}^{-1}$ was reported to have no effect on the mean arterial pressure (21), was also included in the study. Effect of the mentioned drugs on described parameters was also studied during seizures induced by pentylenetetrazole (PTZ).

\section{EXPERIMENTAL}

\section{Chemicals}

L-Arg, L-NAME, 7-NI, AAOA, GABase [a mixture of GABA-T and succinic semialdehyde dehydrogenase (SSDH, EC 1.2.1.16) from Pseudomonas fluorescens] and PTZ were purchased from Sigma Chemical Co. (USA). Standard analytical grade laboratory reagents were obtained from Merck (Germany) or Sigma-Aldrich Chemical Co. (USA). 


\section{Animals}

Experiments were conducted in compliance with the Helsinki Guide for the Care and Use of Laboratory Animals, adopted and promulgated by the EU Directive 2010/63/EU for animal experiments and approved by the institutional committee of ethics of E.N.C.B. (Escuela Nacional de Ciencias Biológicas, Ciudad de México, México).

CD1 male albino mice with the mean body mass of $25 \mathrm{~g}$ (aged 5-6 weeks) were used in the experiments. Animals were maintained under a $12 / 12 \mathrm{~h}$ light/dark cycle, fed ad libitum a stock laboratory diet (\%, $\mathrm{m} / \mathrm{m}: 49.8$ carbohydrates, 23.5 protein, 3.7 fats, minerals and added vitamins and amino acids) and had free access to drinking water.

\section{Brain GABA-T activity determination}

Tissue processing. - After appropriate treatment, animals were sacrificed and whole wet brains were removed. Homogenates were prepared in $5 \%$ Triton X-100 solution ( $25 \%$, $\mathrm{m} / \mathrm{V}$ ) in a tissue homogenizer (Glas-Col, USA). After centrifugation at 12,500 rpm for 45 min, GABA-T activity was determined in the supernatants.

Enzymatic activity evaluation. - GABA-T activity quantification was performed according to the method of Jung et al. (22) using $6 \mu \mathrm{mol} \mathrm{L}^{-1} \mathrm{GABA}$ plus $5 \mu \mathrm{mol} \mathrm{L}{ }^{-1}$ 2-oxoglutarate as substrate. Enzymatic activity was proportional to the formation of NADH from NAD, which was recorded as the increase in absorbance at $340 \mathrm{~nm}$. Specific GABA-T activity was calculated by subtracting the blank values (measured in the absence of substrate) from the total activity. The anticonvulsant AAOA was employed as a control in all cases.

\section{In vitro effect of $N O$ on brain $G A B A-T$ activity}

To the supernatants obtained from mouse brain homogenates, different concentrations of $\mathrm{L}-\mathrm{Arg}\left(10^{-5}-10^{-3} \mathrm{~mol} \mathrm{~L}-1\right)$, L-NAME $\left(10^{-4}-10^{-3} \mathrm{~mol} \mathrm{~L}^{-1}\right)$, 7-NI $\left(10^{-5}-10^{-3} \mathrm{~mol} \mathrm{~L}^{-1}\right)$ or AAOA $\left(10^{-3} \mathrm{~mol} \mathrm{~L}^{-1}\right)$ were added. Several minutes afterwards (10, 15, 20 or $\left.30 \mathrm{~min}\right)$, GABAT activity was determined. Enzymatic activity was compared to that of homogenates from animals that did not receive any treatment.

\section{Ex vivo effect of $N O$ on GABA-T activity}

Groups of 5 animals each received different doses of L-Arg (30-250 $\left.\mathrm{mg} \mathrm{kg}^{-1}\right)$, L-NAME (10-200 $\left.\mathrm{mg} \mathrm{kg}^{-1}\right)$, 7-NI (10-50 $\left.\mathrm{mg} \mathrm{kg}^{-1}\right)$ or AAOA (20-50 $\left.\mathrm{mg} \mathrm{kg}^{-1}\right)$ via i.p. Animals were sacrificed one hour after administration, brains were quickly removed and GABA-T activity was determined.

In a different set of experiments, L-Arg $\left(50 \mathrm{mg} \mathrm{kg}^{-1}\right)$, L-NAME (50 and $\left.100 \mathrm{mg} \mathrm{kg}^{-1}\right)$, 7-NI $\left(10 \mathrm{mg} \mathrm{kg}^{-1}\right)$ or AAOA ( $\left.40 \mathrm{mg} \mathrm{kg}^{-1}\right)$ were i.p. administrated to groups of 5 animals each. After 1 hour of exposure to the respective agent, animals received PTZ $\left(95 \mathrm{mg} \mathrm{kg}^{-1}\right)$. Twenty minutes later, animals were sacrificed and GABA-T activity was determined. Similar experiments were performed at the same time under the same conditions, but without PTZ administration.

In all cases, enzymatic activity was compared to that of animals that received only saline solution. 


\section{Determination of brain GABA concentration}

Tissue processing. - Animals were sacrificed and whole brains were removed. Homogenates in $80 \%$ ethanol were obtained with a Glas-Col tissue homogenizer. Homogenates were centrifuged (3,500 rpm/5 min), pellets were washed in $75 \%$ ethanol and centrifuged at $5000 \mathrm{rpm}$ for $5 \mathrm{~min}$. Supernatants were brought together and chloroform was added. After vigorous shaking and centrifugation at 3,000 rpm for $20 \mathrm{~min}$, the aqueous phase was extracted and concentrated. Aliquots of this sample were used for GABA quantification.

Enzymatic quantification of GABA. - GABA quantification was performed with GABase according to the manufacturer's instructions (Sigma Chemical Co). Briefly, in this method GABA-T converts GABA to succinate semialdehyde (SSA). SSA is then converted to succinate in a reaction catalyzed by $\mathrm{SSDH}$, using $\mathrm{NAD}(\mathrm{P})^{+}$as a cofactor. Stoichiometric reduction of the cofactor, resulting in the increment of the absorbance at $340 \mathrm{~nm}$, reflects the levels of GABA in the reaction mixture. Reaction medium was composed of pyrophosphate buffer $\mathrm{pH}=8.6$, NADP $0.004 \mathrm{~mol} \mathrm{~L}^{-1}, \mathrm{pH}=7$, GABA solution and alpha ketoglutaric acid $0.02 \mathrm{~mol} \mathrm{~L}^{-1}, \mathrm{pH}=7.9$. AAOA was used as a control in all experiments.

\section{Ex vivo effect of $N O$ on GABA brain levels}

Different doses of L-Arg (30-250 $\left.\mathrm{mg} \mathrm{kg}^{-1}\right)$, L-NAME (10-200 mg kg-1), 7-NI (10-50 mg $\mathrm{kg}^{-1}$ ) or AAOA (20-50 mg kg-1) were i.p. administrated to groups of 5 animals each. Animals were sacrificed after 1 hour, the brains were quickly removed and GABA levels were quantified.

In all cases, GABA concentration was compared with the GABA concentration in animals of the control group, which received only saline solution.

\section{Statistical analysis}

All results were normalized against the control and expressed as the mean \pm SEM (standard error of the mean) of at least five determinations $(n>5)$. GABA-T activity was compared between groups using the one-way analysis of variance (ANOVA). F-value was

a) L-Arg

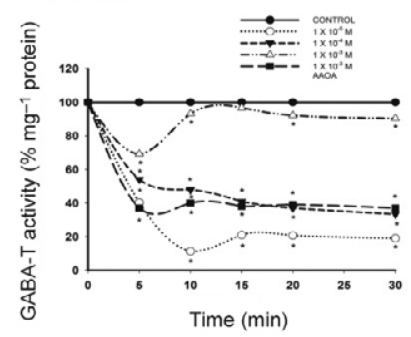

b) L-NAME

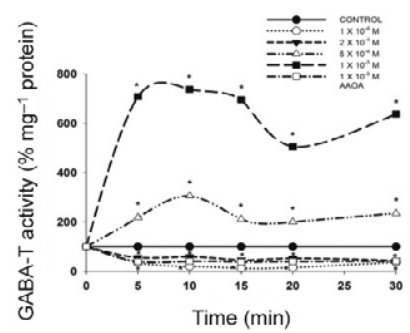

c) $7-\mathrm{NI}$

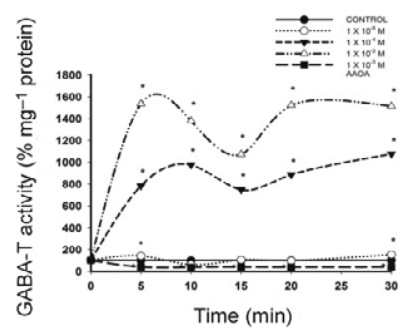

Fig. 1. In vitro influence of some nitric oxide synthase (NOS) effectors on mouse brain gamma amino butyric acid transaminase (GABA-T) activity. a) L-arginine (L-Arg), b) N $\omega$-nitro-L-arginine methyl ester (L-NAME), c) 7-nitroindazole (7-NI) and amino-oxyacetic acid (AAOA). Results are mean \pm SEM $(n>4)$. ${ }^{*}$ Significantly different compared to the control: $p<0.05$. 
calculated and, if and where indicated, followed by Tukey's multiple comparison test. Graph Pad Prism version 5.0 software (GraphPad Software, Inc, La Jolla, CA, USA) was used, and $p<0.05$ was considered statistically significant.

\section{RESULTS AND DISCUSSION}

The aim of this work was to investigate the effect of NO on GABA-T activity and brain GABA levels in order to find out why moderate and high increases in NO levels had opposite effects on GABA-T activity, as reported by Paul et al. (18). NO levels were modified by influencing the NOS activity with its natural substrate (L-Arg) and with unspecific (L-NAME) and neuronal NOS specific (7-NI) inhibitors of its activity, and the resulting changes in GABA-T activity and GABA levels were analyzed in vitro as well as ex vivo. AAOA, a traditional GABA-T inhibitor, was used as a positive control.

a)

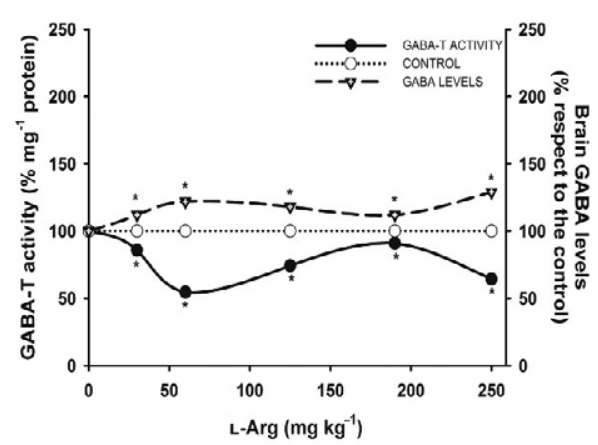

c)

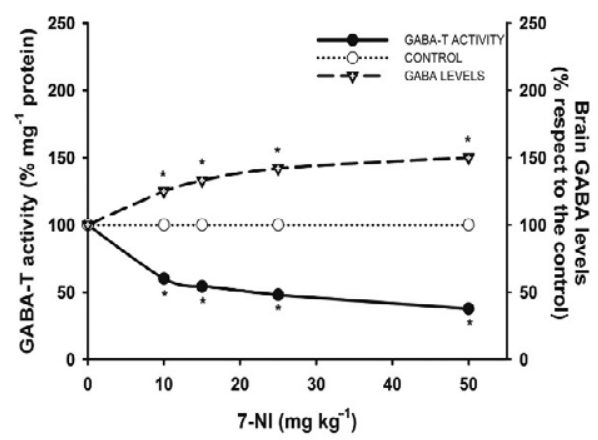

b)

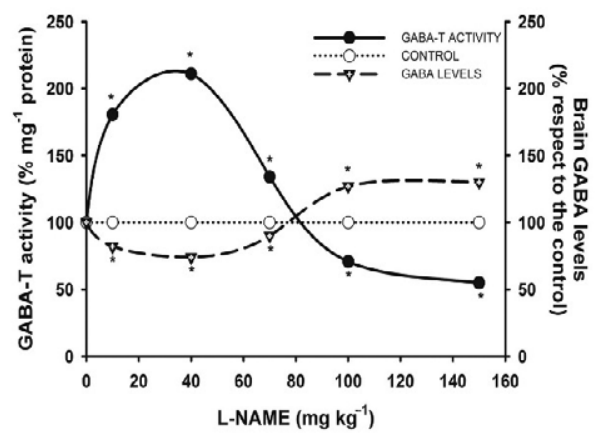

d)

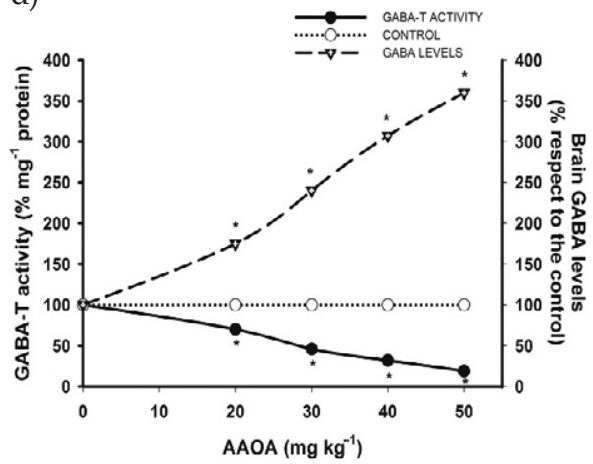

Fig. 2. Ex vivo influence of some nitric oxide synthase (NOS) effectors on mouse brain gamma amino butyric acid transaminase (GABA-T) activity and brain GABA levels. a) L-arginine (L-Arg, 30-250 $\mathrm{mg} \mathrm{kg}^{-1}$ ), b) N $\omega$-nitro-L-arginine methyl ester (L-NAME, 10-200 mg kg-1), c) 7-nitroindazole (7-NI, $10-50 \mathrm{mg} \mathrm{kg}^{-1}$ ) or d) amino-oxyacetic acid (AAOA, 20-50 $\mathrm{mg} \mathrm{kg}^{-1}$ ). Results are mean \pm SEM $(n>4)$. *Significantly different compared to the control: $p<0.05$. 
L-Arg, $10^{-3} \mathrm{~mol} \mathrm{~L}^{-1}$, significantly decreased GABA-T activity in a dose dependent manner in vitro, reaching an activity of only $10 \%$ compared to the control (Fig. 1a). L-NAME increased GABA-T activity in a dose response manner up to $700 \%$ compared to the control (Fig. 1b). The described in vitro variations of GABA-T activity induced by changes in NO levels indicate a clear correlation between these parameters, and are in accord with the reports of Jayakumar et al. (17) and Paul et al. $(18,23)$.

Considering only the results for L-NAME, it could be presumed that an in vitro induced decrease in NO levels caused an increase in GABA-T activity. However, since LNAME is a non-specific NOS inhibitor with an affinity for eNOS of $63-75 \%$ and only of 7 $\%$ for nNOS, its high concentration would be necessary to inhibit the latter NOS isoform. In other words, the role of nNOS is difficult to determine using L-NAME. Thus, a more selective inhibitor of this NO isoform, 7-NI, was used.

Similarly as L-NAME, 7-NI enhanced GABA-T activity but by a much bigger amount, reaching $1.500 \%$ of activity at $10^{-4} \mathrm{~mol} \mathrm{~L}^{-1}$ compared to the control group (Fig. 1c). An about 15 -fold rise of enzymatic activity compared to the control suggests the important role of nNOS in the modulation of GABA-T activity. Considering that high concentrations of LNAME had effects similar to low 7-NI concentrations, the effects of high concentrations of L-NAME on GABA-T activity could be attributed to the inhibition of nNOS.

Fig. 2 represents ex vivo effects of the different NOS effectors. It could be presumed that L-Arg decreased GABA-T activity at two different stages, represented by two different picks. First, NO levels decreased by $40-50 \%$ at a dose of $60 \mathrm{mg} \mathrm{kg}^{-1}$, returning slowly to the control levels at a dose of $190 \mathrm{mg} \mathrm{kg}^{-1}$. In a second peak or stage, GABA-T activity dropped once again by $40-50 \%$ at a dose of $250 \mathrm{mg} \mathrm{kg}^{-1}$ (Fig. 2a). Therefore, it could be taken that the diminished GABA-T activity generated by NO is observed not only in vitro but also ex vivo, but showing two stages, which will be called biphasic. This biphasic shape may represent the effect of NO generated by different NOS isoforms, implying different effects of NO on GABA-T activity, which could depend on whether it is generated by eNOS or nNOS (location dependent).

The former hypothesis seems to be enforced by the effects of different doses of LNAME (Fig. 2b), which also shows two peaks, reaching an increase of $225 \%$ compared to the control $\left(40 \mathrm{mg} \mathrm{kg}^{-1}\right)$, whereas higher doses decreased the activity, reaching a $50 \%$ activity compared to the control.

As regards the effects of 7-NI ex vivo, it decreased GABA-T in a dose dependent manner, reaching about $40 \%$ of enzymatic activity compared to the control and showing only one peak (Fig. 2c). Considering the specific inhibition of 7-NI on nNOS and that its inhibition on GABA-T activity did not show a biphasic effect, along with the fact that 7-NI decreased enzymatic activity by a similar magnitude to that observed at the higher tested dose of L-NAME, it is proposed that the increase of GABA-T activity may be attributed to the inhibition of eNOS, whereas the diminution of the enzymatic activity could be considered as a result of nNOS inhibition.

Overall, ex vivo results suggest that endothelial and neuronal NO have opposite effects on GABA-T activity and their effect as an activator or inhibitor of the enzyme may be related to their anticonvulsant or proconvulsant properties. Therefore, NO brain levels may constitute a new mechanism for GABA-T activity regulation, modulating different pathways and suggesting a possible dependence on location (in the glia or in neurons). This hypothesis, however, needs further investigation. 
In vitro results for 7-NI are opposite to those observed in vivo, since it caused an increase but also a decrease of GABA-T activity, resp. In vitro experiments were designed to study the direct effect of drugs on GABA-T activity without the influence of any other factor, whereas in vivo studies showed the effect on enzymatic activity as a result of integral effects of drugs on the different body systems. Thus, the contrast between in vitro and in vivo effects of 7-NI is considered to be the result of its effect not only on the enzyme but also on other systems that may influence GABA-T activity. Since brain GABA-T is the subject of this study, the effects of NO on the nervous system, which may have repercussions on GABA-T activity, are of particular interest. For example, it has been reported that NO evokes the release of several neurotransmitters, including $\operatorname{GABA}(7,24)$.

Considering the forgoing and the role of GABA-T on the regulation of GABA brain levels, L-Arg, L-NAME and 7-NI effects on brain GABA levels were also investigated. L-Arg increased brain GABA levels, showing also two different peaks (Fig. 2a). This means that an increase in NO levels induced by L-Arg induced, in turn, an increase of brain GABA levels, in a biphasic process corresponding to the two stages of the effect of NO on GABA$\mathrm{T}$ activity. This is in accord with the findings of several authors $(7,15,16)$. The fact that sodium nitroprusside, another NO donor, also increased brain GABA levels (18) supports the idea that NO could modulate the GABA-ergic system.

The effect of L-NAME on brain GABA levels show also two peaks, diminishes with the dose up to $75 \mathrm{mg} \mathrm{kg}^{-1}$ but rises with $100 \mathrm{mg} \mathrm{kg}^{-1}$ or higher (Fig. 2b). This is in agreement with the biphasic effect of L-NAME on GABA-T activity and with the biphasic modulation of GABA release by NO from rat hippocampus reported by Getting et al. (25).

7-NI increased brain GABA levels in a dose dependent manner (Fig. 2c) to a value similar to the highest dose of L-NAME (Fig. 2c), but showed only one peak. The described increase in GABA levels may explain the enhanced anticonvulsant effects of conventional antiepileptic drugs such as carbamazepine, phenobarbital, phenytoin and valproate induced by 7-NI, obtained in maximal electroshock-induced seizures in a mouse model (26).

Altogether, the described effects on brain GABA levels confirm that NO promotes the release of GABA and suggest that this happens through the changes in GABA-T activity. Besides, considering the results for L-NAME and 7-NI and their affinity for eNOS and nNOS, resp., it could be said that neuronal and endothelial NO have opposite effects not only on GABA-T activity but also on brain GABA levels.

Opposite effects of NO, depending on whether eNOS or nNOS is involved, may explain its anticonvulsant but also proconvulsant properties, as reported in different studies (4). Considering that NO as well as GABA-T activity are related to seizures, the possible repercussions of the changes of NO levels on GABA-T activity during convulsions were also studied, investigating the effect of L-Arg, L-NAME and 7-NI on GABA-T activity during seizures induced by PTZ.

PTZ did not modify GABA-T activity by itself but the decrease of GABA-T activity caused by $50 \mathrm{mg} \mathrm{kg}^{-1}$ of L-Arg as well as the increase produced by $50 \mathrm{mg} \mathrm{kg}^{-1}$ of L-NAME were partially counteracted during the seizures induced by PTZ. L-NAME $150 \mathrm{mg} \mathrm{kg}^{-1}$ and 7-NI $10 \mathrm{mg} \mathrm{kg}^{-1}$ decreased enzymatic activity by a similar value (about $60 \%$ ), the effect that was not modified by PTZ (Fig. 3). The increase in enzymatic activity induced by a low dose of L-NAME and the decrease caused by a high dose of the same drug indicate that also during seizures induced by PTZ, L-NAME had different effects on GABA-T activity and, in turn, on brain GABA levels, which seems to be related to the NOS isoform involved. 


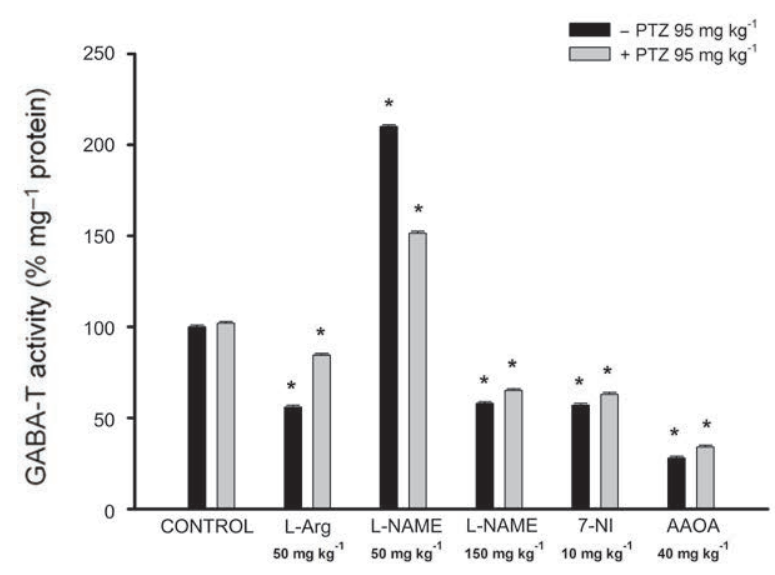

Fig. 3. Ex vivo influence of some nitric oxide synthase (NOS) effectors on mouse brain gamma amino butyric acid transaminase (GABA-T) activity during seizures induced by pentylenetetrazole (PTZ). Animals received L-arginine (L-Arg, $50 \mathrm{mg} \mathrm{kg}^{-1}$ ), N $\omega$-nitro-L-arginine methyl ester (L-NAME, 50 and $150 \mathrm{mg} \mathrm{kg}^{-1}$ ), 7-nitroindazole (7-NI, $10 \mathrm{mg} \mathrm{kg}^{-1}$ ) or amino-oxyacetic acid (AAOA, $40 \mathrm{mg} \mathrm{kg}^{-1}$ ). Seizures were induced by PTZ $\left(95 \mathrm{mg} \mathrm{kg}^{-1}\right)$. Results are mean \pm SEM $(n>4)$. *Significantly different from the group that did not receive PTZ: $p<0.05$.

The primary target of PTZ is to weaken the inhibitory action of GABA, acting as a $\mathrm{GABA}_{\mathrm{A}}$ receptor antagonist. However, seizures induced by this chemoconvulsant are also a result of the activation of glutamatergic synaptic transmission, and NMDA receptors are mainly involved in the genesis of clonic seizures (27). The results reported herein suggest that NO generated by nNOS may be the activator of NMDA receptors that participate in this process, since during seizures induced by PTZ inhibition of nNOS, caused by high doses of L-NAME as well as 7-NI, it decreased GABA-T activity leading to an increase of brain GABA levels. This conclusion is not surprising considering that it has been reported

a) L-Arg

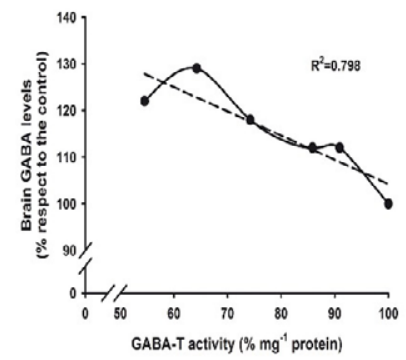

b) L-NAME

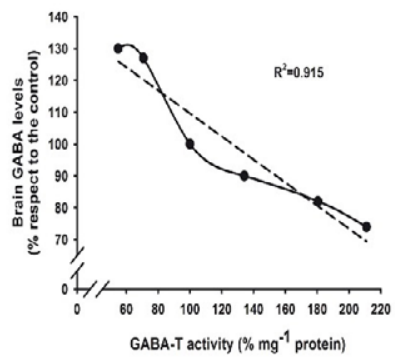

c) 7-NI

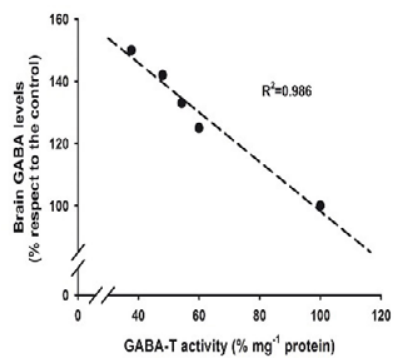

Fig. 4. Correlation between brain gamma amino butyric acid transaminase (GABA-T) activity and brain GABA levels under the influence of L-arginine (L-Arg), N $\omega$-nitro-L-arginine methyl ester (L-NAME) and 7-nitroindazole (7-NI). 
that NMDA receptors/NO pathway contribute to the anticonvulsant effect of different drugs such as D-penicillamine (11), dextrometrophan (28) and lithium (29).

Once established that NO influences the GABA-T activity modulating brain GABA levels, the correlations between GABA-T activity and GABA levels in the presence of LArg, L-NAME and 7-NI were explored. Fig. 4 shows, as expected, that GABA-T activity and brain GABA levels are inversely related, but only in the case of 7-NI (Fig. 4c) the correlation is linear with $R^{2}=0.986$. These results suggest that NO generated by nNOS had a direct influence on GABA-T activity and thereby on brain GABA levels.

\section{CONCLUSIONS}

Contradictory reports on the role of $\mathrm{NO}$ on the seizure mechanism may be explained by the NOS isoform involved. The results confer anticonvulsant properties on endothelial NO but proconvulsant on neuronal NOS. This attribution of convulsant properties to neuronal NO is in agreement with the finding that the anticonvulsant effect of dextrometrophan is mediated by a decline in nNOS activity (28). Proconvulsant or anticonvulsant properties of NO may be explained by the opposite effects of endothelial and neuronal NO on GABA-T activity, and hence on GABA levels.

More work is necessary to assess exactly the role of neuronal $\mathrm{NO}$ and its particular action site, since it has different effects on the brain. For example, it has been reported that nNOS- derived excess NO in the glutamatergic pathway plays a key role in the failure of the blood-brain-barrier during seizures induced by PTZ (30).

Understanding how to modulate brain GABA-T activity through NO brain levels represents a novel strategy for controlling seizures, and hence for the development of new antiepileptic drugs (AEDS), which is especially important in the cases refractory to the treatment with traditional AEDS, either administered alone or in combination.

Acknowledgements. - This work was supported by the Section of Postgraduate Studies and Research, Academic Secretary of National Polytechnic Institute, México.

Acronyms, abbreviations, symbols. - AAOA - amino oxyacetic acid, AEDS - antiepileptic drugs, L-Arg - L-arginine, cGMP - cyclic guanosine monophosphate, CNS - central nervous system, GABA - gamma aminobutyric acid, GABA-T - GABA transaminase, NAD $(P)$ - nicotinamide adenine dinucleotide(phosphate), L-NAME - N-nitro-L-arginine-methyl ester, 7-NI - 7-nitroindazole, NMDA - N-methyl-D-aspartate (NMDA), NO - nitric oxide, NOS - NO synthase (eNOS - endothelial NOS, iNOS - inducible NOS, nNOS - neuronal NOS), PTZ - pentylenetetrazole, SSA - succinate semialdehyde, SSDH - succinic semialdehyde dehydrogenase.

\section{REFERENCES}

1. M. Hoffman, A new role for gases: neurotransmission, Science 28 (1991) 1788-1788; https://doi. org/10.1126/science.1712121

2. S. Moncada, R. M. Palmer and E. A. Higgs, Nitric oxide: physiology, pathophysiology, and pharmacology, Pharmacol. Rev. 43 (1991) 109-142.

3. K. S. Raevskii, V. G. Bashkatova and A. F. Vanin, The role of nitric oxide in brain glutaminergic pathology, Vestn. Ross. Akad. Med. Nauk 1 (2000) 11-15. 
4. M. Banach, B. Piskorska, S. J. Czuczwar and K. K. Borowicz, Nitric oxide, epileptic seizures, and action of antiepileptic drugs, CNS Neurol. Disord. Drug Targets 10 (2011) 808-819; https://doi. org/10.2174/187152711798072347

5. J. Garthwaite and C. L.Boulton, Nitric oxide signaling in the central nervous system, Annu. Rev. Physiol. 57 (1995) 683-706; https://doi.org/10.1146/annurev.ph.57.030195.003343

6. A. Kumar, S. Lalitha and J. Mishra, Possible nitric oxide mechanism in the protective effect of hesperidin against pentylenetetrazole (PTZ)-induced kindling and associated cognitive dysfunction in mice, Epilepsy Behav. 29 (2013) 103-111; https://doi.org/10.1016/j.yebeh.2013.06.007

7. K. Kuriyama and S. Ohkuma, Role of nitric oxide in central synaptic transmission: effects on neurotransmitter release, Jpn. J. Pharmacol. 69 (1995) 1-8; https://doi.org/10.1254/jjp.69.1

8. M. J. Owens and C. B. Nemeroff, Pharmacology of valproate, Psychopharmacol. Bull. 37 (2003) 17-24.

9. Q. P. Wang, F. Jammoul, A. Duboc, J. Gong, M. Simonutti, E. Dubus, C. M. Craft, W. Ye, J. A. Sahel and S. Picaut, Treatment of epilepsy: the GABA-transaminase inhibitor, vigabatrin, induces neuronal plasticity in the mouse retina, Eur. J. Neurosci. 27 (2008) 2177-2187; https://doi. org/10.1111/j.1460-9568.2008.06175.x

10. M. Qume and L. J. Fowler, Effect of chronic treatment with the GABA transaminase inhibitors g-vinyl GABA and ethanolamine O-sulphate on the in vitro GABA release from rat hippocampus, Br. J. Pharmacol. 122 (1997) 539-545; https://doi.org/10.1038/sj.bjp.0701383

11. N. Rahimi, M. Sadeghzadeh, M. Javadi-Paydar, M. R. Heidary, F. Jazaeri and A. R. Dehpour, Effects of D-penicillamine on pentylenetetrazole-induced seizures in mice: involvement of nitric oxide/ NMDA pathways, Epilepsy Behav. 39 (2014) 42-47; https://doi.org/10.1016/j.yebeh.2014.07.013

12. Q. Wang, E. Mergia, D. Koesling and T. Mittmann, Nitric oxide/cGMP signaling via guanylyl cyclase isoform 1 modulates glutamate and GABA release in somatosensory cortex of mice, Neuroscience 30 (2017) 180-189; https://doi.org/10.1016/j.neuroscience.2017.07.063

13. J. P. Pin, B. J. Van-Vliet and J. Bockaert, NMDA-and kainate-evoked GABA release from striatal neurons differentiated in primary culture: differential blocking by phencyclidine, Neurosci. Lett. 87 (1988) 87-92; https://doi.org/10.1016/0304-3940(88)90150-4

14. J. G. Valtschanoff, R. J. Weinberg, V. N. Kharazia, H. H. Schmidt, M. Nakane and A. Rustioni, Neurons in rat cerebral cortex that synthesize nitric oxide: NADPH diaphorase histochemistry, NOS immunocytochemistry, and colocalization with GABA, Neurosci. Lett. 157 (1993) 157-161.

15. G. Lonart, J. Wang and K. M. Johnson, Nitric oxide induces neurotransmitter release from hippocampal slices, Eur. J. Pharmacol. 220 (1992) 271-272; https://doi.org/10.1016/0014-2999(92)90759-W

16. G. Segovia and F. Mora, Role of nitric oxide in modulating the release of dopamine, glutamate, and GABA in striatum of the freely moving rat, Brain Res. Bull. 45 (1998) 275-279; https://doi.org/10.1016/ S0361-9230(97)00402-4

17. A. R. Jayakumar, R. Sujatha, V. Paul, C. Asokan, S. Govindasamy and R. Jayakumar, Role of nitric oxide on GABA, glutamic acid, activities of GABA-T and GAD in rat brain cerebral cortex, Brain Res. 837 (1999) 229-235; https://doi.org/10.1016/S0006-8993(99)01692-3

18. V. Paul and P. Ekambaram, Effects of sodium nitroprusside, a nitric oxide donor, on gamma-aminobutyric acid concentration in the brain and on picrotoxin-induced convulsions in combination with phenobarbitone in rats, Pharmacol. Biochem. Behav. 80 (2005) 363-370.

19. J. Rodrigo, D. R. Springall, O. Uttenthal, M. L. Bentura, F. Abadia-Molina, V. Riveros-Moreno, R. Martinez-Moreno, J. M. Polak and S. Moncada, Localization of nitric oxide synthase in the adult rat brain, Philos. Trans. Royal Soc. London B, Biol. Sci. 345 (1994) 175-221; https://doi.org/ 10.1098/ rstb.1994.0096

20. S. Gotti, M. Sica, C. Viglietti-Panzica and G. Panzica, Distribution of nitric oxide synthase immunoreactivity in the mouse brain, Microsc. Res. Tech. 68 (2005) 13-35.

21. P. K. Moore, P. Wallace, Z. Gaffen, S. L. Hart and R. C. Babbedge, Characterization of the novel nitric oxide synthase inhibitor 7-nitro indazole and related indazoles: antinociceptive and cardiovascular effects, Br. J. Pharmacol. 110 (1993) 219-224; https://doi.org/10.1111/j.1476-5381.1993.tb13795.x 
22. M. J. Jung, B. Lippert, B. W. Metcalf, P. J. Schechter, P. Bohlen and A. Sjoerdsma, The effect of 4-amino hex-5-ynoic acid (gamma-acetylenic GABA, gamma-ethynyl GABA) a catalytic inhibitor of GABA transaminase, on brain GABA metabolism in vivo, J. Neurochem 28 (1977) 717-723; https://doi. org/10.1111/j.1471-4159.1977.tb10618.x

23. V. Paul and A. R. Jayakumar, A role of nitric oxide as an inhibitor of gamma-aminobutyric acid transaminase in rat brain, Brain Res. Bull. 51 (2000) 43-46.

24. J. Orzelska, S. Talarek S, J. Listos and S. Fidecka, Divergent effects of L-arginine-NO pathway modulators on diazepam and flunitrazepam responses in NOR task performance, Behav. Brain Res. 284 (2015) 179-186; https://doi.org/10.1016/j.bbr.2015.02.014

25. S. J. Getting, J. Segieth, S. Ahmad, C. S. Biggs and P. S. Whitton, Biphasic modulation of GABA release by nitric oxide in the hippocampus of freely moving rats in vivo, Brain Res. 717 (1996) 196-199; https://doi.org/10.1016/0006-8993(96)00127-8

26. J. J. Luszczki, A. Sacharuk, A. Wojciechowska, M. M. Andres-Mach, M. Dudra-Jastrzebska, M. Mohamed, K. M. Sawicka, J. Kozinska and S. J. Czuczwar, 7-Nitroindazole enhances dose-dependently the anticonvulsant activities of conventional antiepileptic drugs in the mouse maximal electroshock-induced seizure model, Pharmacol. Rep. 58 (2006) 660-671.

27. N. Y. Lukomskaya, N. I. Rukoyatkina, L. V. Gorbunova, V. E. Gmiro and L. G. Magazanik, Studies of the roles of NMDA and AMPA glutamate receptors in the mechanism of corasole convulsions in mice, Neurosci. Behav. Physiol. 34 (2004) 783-789; https://doi.org/10.1023/B:NEAB.0000038128.02725.7e

28. G. Mohseni, S. Ostadhadi, R. Akbarian, M. Chamanara, A. Norouzi-Javidan and A. R. Dehpour, Anticonvulsant effect of dextrometrophan on pentylenetetrazole-induced seizures in mice: Involvement of nitric oxide and N-methyl-D-aspartate receptors, Epilepsy Behav. 65 (2016) 49-55; https:// doi.org/10.1016/j.yebeh.2016.08.001

29. R. M. Jafari, M. H. Ghahremani, N. Rahimi, A Shadboorestan, A. Rashidian, J. Esmaeili, S. Ejtemaei Mehr and A. R. Dehpour, The anticonvulsant activity and cerebral protection of chronic lithium chloride via NMDA receptor/nitric oxide and phospho-ERK, Brain Res. Bull. 137 (2017) 1-9; https:// doi.org/10.1016/j.brainresbull.2017.10.015

30. S. Danjo, Y. Ishihara, M. Watanabe, Y. Nakamura and K. Itoh, Pentylentetrazole-induced loss of blood-brain barrier integrity involves excess nitric oxide generation by neuronal nitric oxide synthase, Brain Res. 1530 (2013) 44-53; https://doi.org/10.1016/j.brainres.2013.06.043 